\title{
Saúde mental e o isolamento social de idosos em período de pandemia
}

RESUMO | Objetivo: Descrever impactos que o isolamento social pode acarretar na saúde mental dos idosos durante a pandemia. Método: Trata-se de uma revisão bibliográfica integrativa, utilizando os descritores "Coronavirus Infections ", "Pandemics", "Mental Health ", e "Aged", realizando a combinação entre si e o operador boleano OR como estratégia de busca nas bases de dados: LILACS, SciELO, WEB OF SCIENCE. Adotados critérios de inclusão: publicações no período de 2020 que tratassem do assunto, em português e inglês. Como critérios de exclusão: repetição nas bases de dados, artigos fora do contexto quanto a pergunta norteadora da pesquisa. Resultado: Foram encontrados 32 artigos, selecionados 26, e destes foram analisados 08. Conclusão: A saúde mental em período de pandemia foi explícita como um fator de impacto, refletindo os desfechos favoráveis e desfavoráveis no contexto da saúde mental. É evidente a necessidade de promoção para intervenções afim de minimizar impactos negativos para a saúde mental.

Palavras-chaves: Infecções por Coronavírus, Pandemias, Saúde Mental, Idoso.

\begin{abstract}
Objective: To describe the impacts that social isolation can have on the mental health of the elderly during the pandemic. Method: This is an integrative bibliographic review, using the descriptors "Coronavirus Infections", "Pandemics", "Mental Health", and "Aged", making the combination between you and the Boolean operator OR as a search strategy in the databases data: LILACS, SciELO, WEB OF SCIENCE. Adopted inclusion criteria: publications in the period of 2020 that dealt with the subject, in Portuguese and English. As exclusion criteria: repetition in the databases, articles out of context regarding the guiding question of the research. Result: 32 articles were found, selected 26, of which 8 were analyzed. Conclusion: Mental health in a pandemic period was explicit as an impact factor, reflecting favorable and unfavorable outcomes in the context of mental health. The need for promotion for interventions is evident in order to minimize negative impacts on mental health.
\end{abstract}

Keywords: Coronavirus Infections, Pandemics, Mental Health, Aged.

RESUMEN | Objetivo: Describir los impactos que el aislamiento social puede tener en la salud mental de los ancianos durante la pandemia. Método: Esta es una revisión bibliográfica integradora, utilizando los descriptores "Infecciones por coronavirus", "Pandemias", "Salud mental" y "Envejecido", haciendo la combinación entre usted y el operador booleano O como una estrategia de búsqueda en las bases de datos. datos: LILACS, SCiELO, WEB OF SCIENCE. Criterios de inclusión adoptados: publicaciones en el período de 2020 que trataron el tema, en portugués e inglés. Como criterios de exclusión: repetición en las bases de datos, artículos fuera de contexto sobre la cuestión orientadora de la investigación. Resultado: Se encontraron 32 artículos, se seleccionaron 26, de los cuales se analizaron 8. Conclusión: La salud mental en un período de pandemia fue explícita como factor de impacto, reflejando resultados favorables y desfavorables en el contexto de la salud mental. La necesidad de promover intervenciones es evidente para minimizar los impactos negativos en la salud mental.

Palavras claves: Infecciones por Coronavirus, Pandemias, Salud Mental, Ancioano.

\section{Jaqueline Maria Silva dos Santos}

Graduanda do Curso de enfermagem do Centro Universitário Mário Pontes Jucá - AL. ORCID: 0000-0003-3690-7811

\section{Euda Maria dos Santos Messias}

Graduanda do Curso de enfermagem do Centro Universitário Mário Pontes Jucá -AL. ORCID: 0000-0003-3073-2686

\section{Raquel Ferreira Lopes}

Professor Orientador: Mestre, Centro Universitário Mário Pontes Jucá- AL. ORCID: 0000-0002-2061-7038

INTRODUÇÃO

$\Lambda$ tualmente uma das maiores emergências de saúde pública do mundo é o novo Coronavírus (COVID-19). Em dezembro de 2019 foi registrado o primeiro caso de infecção na China ${ }^{1}$. Essa emergência em saúde, está fazendo com que aumentem os desafios para cuidarmos dos mais velhos seja na comunidade, nos lares de idosos ou até mesmo nos hospitais ${ }^{2}$.

Durante a pandemia por Coronavírus, em que os idosos devem permanecer em isolamento, é importante que cuidadores e familiares fiquem atentos ao primeiro sinal de tristeza, desânimo, falta de energia, pensamentos negativos, falta de esperança e mudanças no comportamento. Esse fardo acarreta em angústia e a sentimentos desfavoráveis como a frustração e tristeza que são prejudiciais à manutenção da saúde mental da pessoa idosa ${ }^{3}$. Evidencia-se ainda que esses conflitos podem apresentar como consequência o isolamento e a solidão ${ }^{4}$.

No entanto o isolamento social é algo importante quando se fala no contexto em saúde, pessoas que se encontram em situação de solidão excessiva, são mais dispostas a depressão e a outros transtornos mentais, muitas vezes caracteriza-se pelo humor deprimido e pela perda de interesse em várias atividades 5 . Há lacunas no diagnóstico e tratamento adequado, bem como insuficientes ações adotadas e destinadas à promoção da saúde mental da população em discussão . $^{6}$

Devido ao grande número de incidência, afim de minimizar as consequências 
causadas pelo COVID-19, alguns países têm adotado medidas de controle como o isolamento social de escolas, creches, idosos, pessoas do grupo de risco e a quarentena de toda população ${ }^{3}$.

Durante a pandemia a diminuição da dinâmica que muitos exerciam acabou sendo prejudicada como visitar os filhos, netos, parentes mais próximos e as reuniões em família. No entanto salienta-se que durante esse período deva existir um olhar de forma mais voltado para o idoso de familiares e amigos, visto que doenças psíquicas podem ser agravadas podendo levar a transtornos de pânico ou até mesmo o suicídio.

Diante do exposto esse estudo buscará responder a seguinte pergunta norteadora da pesquisa quais os aspectos que o isolamento social pode acarretar na saúde mental dos idosos durante a pandemia? Logo o objetivo geral é descrever os aspectos da saúde mental e o isolamento social dos idosos em período de pandemia através de uma investigação na literatura científica disponível.

\section{MÉTODO}

Trata-se de um estudo de revisão integrativa da literatura seguindo a metodologia orientada por Mendes (2008) que a contempla em seis fazes de construção: identificação do tema e seleção da hipótese, busca na literatura, categorização dos estudos, avaliação dos estudos incluídos, interpretação dos resultados, apresentação da revisão. E afirma também que a contribuição da síntese dos resultados de pesquisas na incorporação de evidências produz conhecimento novo para a prática ${ }^{7}$.

Logo, a primeira etapa deste estudo que delimita o problema de pesquisa já foi descrita no decorrer da introdução, favorecendo a compreensão lógica do processo de elaboração.

A busca na literatura e o período de coleta de dados aconteceu em junho de 2020 e identificou os artigos acerca deste assunto através da utilização dos descritores e MeSh: "Coronavirus Infections ", "PandemiCs", "Mental Health ", e "Aged", realizando a combinação entre si com o recurso do operador boleano OR como estratégia de busca nas bases de dados: Literatura Latino-americana e do Caribe em Ciências da Saúde (LILACS), Scientific Electronic Library Online (SciELO) e Web of Science.

O filtro inserido nesta busca corresponde ao período de publicação relacionado ao ano de 2020 devido a ampla disponibilidade da literatura nesse período, nos idiomas Inglês e português. Sendo excluídos: repetição nas bases de dados, artigos fora do contexto e ausência de resposta quanto a pergunta norteadora da pesquisa.

Para categorizar os estudos optou-se pela extração das seguintes informações: título, autores, percurso metodológico, amostra, país, periódico e ano de publicação, e as considerações relacionadas ao tema central do objeto de discussão desta revisão. Nos casos em que o resumo era considerado suficiente, selecionavam-se os artigos e a versão integral era obtida para confirmar a elegibilidade e assim incluir no estudo.

As etapas de avaliação dos estudos e de interpretação dos resultados foram realizadas de forma impessoal, revisada em pares, detalhada de forma criteriosa, buscando a compreensão dos resultados, discutindo a associação dos mesmos com

Figura 1: Fluxograma de identificação e seleção dos artigos para revisão integrativa sobre a saúde mental dos idosos em período de pandemia, 2020.

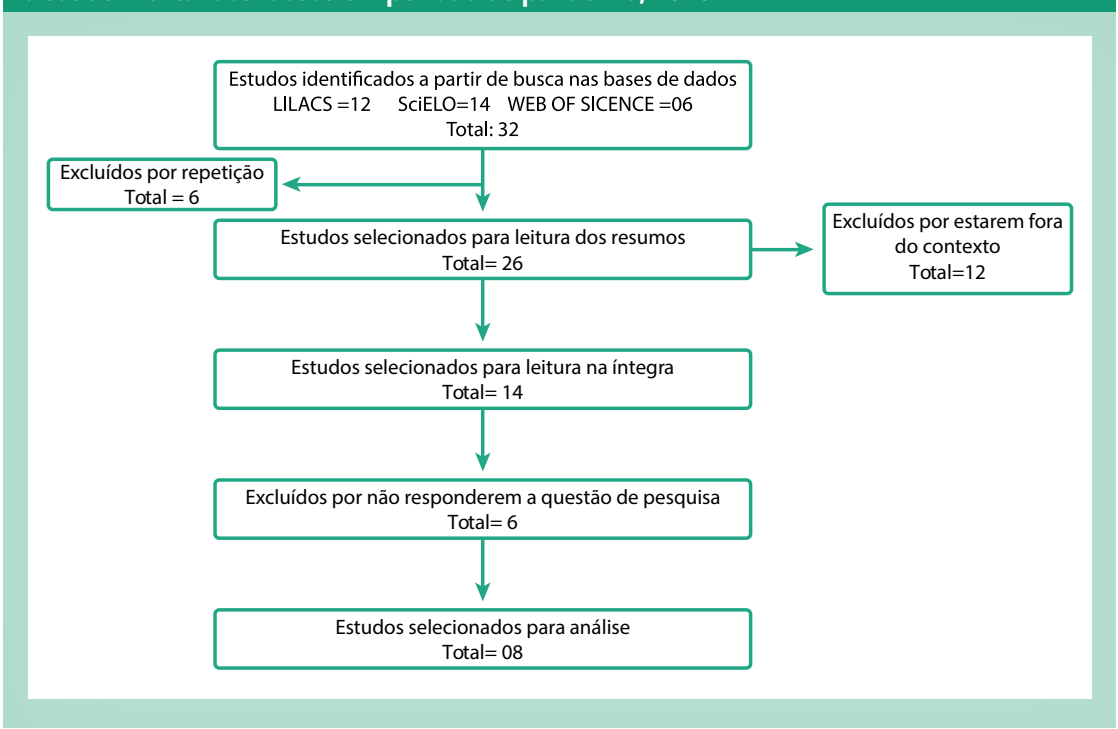

Fonte: Dados da própria pesquisa, 2020. os aspectos relacionados a resposta da pergunta norteadora desta revisão.

Seguindo para última etapa que é a descrição da síntese da revisão na constituição deste estudo aqui apresentado.

\section{RESULTADOS}

Este estudo teve como prioridade extrair da literatura cientifica informações relevantes que pudessem categorizar a relação entre saúde mental e a saúde do idoso durante a pandemia.

Para facilitar o entendimento dos resultados obtidos foi elaborado um fluxograma com os passos percorridos no decorrer da seleção dos artigos sendo exibido através da Figura1.

Então, foi demonstrado na Figura 1 a busca inicial de dados que possibilitou a identificação de artigos na base de dados LILACS 12, SciELO 14 e Web of Science 06. Resultando em 32 estudos encontrados através da estratégia de busca delimitada e com a inserção de filtros.

A matrix de síntese desta revisão integrativa, exibida na Tabela 1. Descreve aspectos predominantes na produção do conhecimento sobre a relação do idoso e sua saúde mental frente a uma situação de pandemia. 
Tabela 1: Matriz de sintese dos artigos sobre sobre a saúde mental dos idosos em período de pandemia, 2020.

\begin{tabular}{|c|c|c|c|c|}
\hline Artigo & Título e Autores & $\begin{array}{l}\text { Percurso Me- } \\
\text { todológico }\end{array}$ & Amostra e País & Periódico e Ano \\
\hline 1 & $\begin{array}{l}\text { Mood Disorders in Late Life: A Population-based Analysis of } \\
\text { Prevalence, Risk Factors, and Consequences in Community- } \\
\text {-dwelling Older Adults in Ontario. Strauss R.; Kurdyak P. }\end{array}$ & $\begin{array}{l}\text { Estudo } \\
\text { transversal }\end{array}$ & $\begin{array}{l}\text { Indivíduos de } 65 \text { anos ou mais. } \\
\text { Ontario }\end{array}$ & $\begin{array}{l}\text { The Canadian Jour- } \\
\text { nal of Psychiatry. } \\
2020\end{array}$ \\
\hline 2 & $\begin{array}{l}\text { Israel Ad Hoc COVID-19 Committee: Guidelines for Care } \\
\text { of Older Persons During a Pandemic. Clarfield et al. }\end{array}$ & $\begin{array}{l}\text { Validação de } \\
\text { Instrumento }\end{array}$ & $\begin{array}{l}\text { Idosos na comunidade e em Institui- } \\
\text { ções de Longa Permanência. Israel. }\end{array}$ & $\begin{array}{l}\text { The American Geria- } \\
\text { trics Society. } 2020 .\end{array}$ \\
\hline 4 & $\begin{array}{c}\text { Perfil de saúde mental de idosos comunitários: um estudo } \\
\text { transversal. Cordeiro et al. }\end{array}$ & $\begin{array}{l}\text { Estudo } \\
\text { transversal }\end{array}$ & $\begin{array}{l}\text { Idosos cadastrados na área de abran- } \\
\text { gência de três equipes de saúde da } \\
\text { família. Recife }\end{array}$ & $\begin{array}{l}\text { Rev Bras Enferm. } \\
2020 .\end{array}$ \\
\hline 5 & $\begin{array}{c}\text { Fatores associados a sintomas depressivos em idosos } \\
\text { inseridos em contexto de vulnerabilidade social. } \\
\text { Didone et al. }\end{array}$ & $\begin{array}{l}\text { Estudo } \\
\text { transversal }\end{array}$ & $\begin{array}{c}\text { Estudo transversal realizado com } 302 \\
\text { idosos de comunidade cadastrados } \\
\text { em Unidades de Saúde da Família. } \\
\text { NASF São Carlos }\end{array}$ & $\begin{array}{l}\text { Rev Bras. En- } \\
\text { ferm.2020. }\end{array}$ \\
\hline 6 & $\begin{array}{l}\text { Sintomas somáticos, sintomatologia depressiva e ansió- } \\
\text { gena em pessoas idosas. Gonçalves et al. }\end{array}$ & $\begin{array}{l}\text { Quantitativo e } \\
\text { transversal. }\end{array}$ & $\begin{array}{l}91 \text { pessoas idosas, com uma média } \\
\text { de idades de } 78,40 \text {. Portugal. }\end{array}$ & $\begin{array}{l}\text { Psicologia, saúde \& } \\
\text { doenças, } 2020\end{array}$ \\
\hline 7 & $\begin{array}{l}\text { Nursing homes and the elderly regarding the COVID-19 } \\
\text { pandemic: situation report from Hungary. Kemenesi et al. }\end{array}$ & $\begin{array}{l}\text { Relato de } \\
\text { situação }\end{array}$ & $\begin{array}{l}\text { Dados epidemiológicos coletados do } \\
\text { banco de dados da Academia Húnga- } \\
\text { ra de Ciência. Hungria. }\end{array}$ & GeroScience. 2020. \\
\hline 8 & $\begin{array}{c}\text { Prevalência de sintomatologia depressiva em idosos } \\
\text { brasileiros: uma revisão sistemática com metanálise. } \\
\text { Meneguci et al. }\end{array}$ & $\begin{array}{l}\text { Revisão siste- } \\
\text { mática com } \\
\text { metanálise }\end{array}$ & $\begin{array}{l}\text { Estudos com idosos brasileiros que } \\
\text { identificaram a prevalência de sinto- } \\
\text { matologia depressiva. Brasil. }\end{array}$ & $\begin{array}{l}\text { J Bras Psiquiatr. } \\
2019 .\end{array}$ \\
\hline
\end{tabular}

Fonte: Dados da própria pesquisa, 2020.

Tabela 2: Detalhamento dos achados sobre a saúde mental dos idosos em periodo de pandemia.

\section{Artigo Principais achados}

A proporção dos homens é maior que vivem sozinhos, e uso crônico de opióide,

1 transtorno de humor maiores nas mulheres interferindo na saúde mental favorecendo o surgimento de doenças psicopatológicas.

2 Promover diretrizes para triagem de idosos com infecção por COVID-19, estratégias poderá resultar diminuição do risco de doenças relacionadas a saúde mental.

Presença de sintomatologia psicopatológica nos participantes. Sendo a interven-

3 ção psicológica um método eficaz na sua redução, salienta-se a pertinência da avaliação e intervenção psicológica em instituições de apoio a idosos.

4 Foi possível identificar desencadeadores de sofrimento psíquico, auxiliando a equipe de enfermagem no desenvolvimento de ações preventivas e de cuidado.

Qualidade de vida e suporte social foram identificados como fatores protetores

5 para sintomas depressivos, enquanto estar sob risco de desnutrição, morar sozinho, relatar dor e ser do sexo feminino são fatores preditores.

6 Avaliação e compreensão das queixas somáticas de idosos, se considerarem sintomas psicológicos, nomeadamente, a sintomatologia depressiva.

Enfatizam a necessidade urgente de autoridades de saúde pública se concentrem

7 nos lares de idosos e unidades de serviços residenciais em todo o mundo devido a vulnerabidade dos idosos em contrairem a infecção por COVID-19.

8 Estratégias de intervenção para reduzir a prevalência de sintomatologia depressiva.

Fonte: Dados da própria pesquisa, 2020.

\section{DISCUSSÃO}

Alguns aspectos podem acabar contribuindo negativamente para a terceira idade em um período de pandemia, pois é um momento de grandes transformações para o indivíduo principalmente para a pessoa idosa. Isso inclui uma série de mudanças físicas, aposentadoria, doenças, afastamento ou perda de pessoas queridas, além de uma redução da independência e autonomia de modo geral.

Em países com Israel por exemplo os serviços de saúde oferecem serviços gratuitos como os que operam no Canadá e Reino Unido, a medicina no país é bem desenvolvida em serviços para a pessoa idosa. Lá funciona da seguinte forma, se um idoso adoecer principalmente com sintomas por COVID-19 ele é avaliado imediatamente, caso sua condição de saúde permita que ele se trate em casa e assim ele optar ele será tratado em casa , os centros de apoio dos Estados Unidos da América (EUA), oferecem di- 
retrizes senssatas para este fim. Além de tudo é oferecido medicamentos aprovados pelo Ministério da Saúde para os tratamentos orais para COVID- 19, oxigenoterapia domiciliar, e os medicamentos para as outras comorbidades já tão presentes na saúde do idoso².

Consequências a exemplo da depressão que é um dos fatores que mais acometem as mulheres idosas com cerca de 7,1\%, em comparação com os homens 4,9\%. Um transtorno de humor com prevalência geral que diminuiu com o aumento da idade, de $6,9 \%$ nas pessoas de 65 a 74 anos, 5,2\% nas de 75 a 84 anos e 4,2\% nas pessoas de 85 anos ou mais. Alguns dos correspondentes mais fortes de transtornos do humor também incluem uso crônico de opióides, insegurança alimentar, comorbidades e tabagismo ${ }^{8}$.

A clínica do idoso é potencialmente exarcebada em relação ao fenômeno social da descrença, aspectos como a discriminação baseadas na idade cronológica que era como estava sendo observado em muitos países europeus, como Itália, Espanha e França, a modesta capacidade da unidade de terapia intensiva per capita (UTI) de Israel seria sobrecarregada por novos casos, muitos deles pessoas idosas².

Idosos esses que estão no centro da atenção quando se fala de coronavírus, pois em meio a medidas de precaução como o isolamento social e muitas informações circulando algumas verdadeiras e outras falsas, as pessoas idosas acabam ficando privadas de seu convívio social trazendo em excesso a ansiedade o medo e angústia.

Outro aspecto importante são os lares de idosos fortemente afetados, foi observado anteriormente à pandemia atual que as populações idosas que vivem em asilos ou lares de idosos têm papel predominante nas epidemias, uma vez que as circunstâncias em um lar de idosos proporcionam um ambiente ideal para a aquisição de infecção, os residentes são suscetíveis pois compartilham fontes de ar, alimentos, água e saúde cuidados em um ambiente institucionalizado lotado 9 .

Então é essencial que medidas simples de rotinas diária tentem se manter, como não fazer com que os idosos se sintam inúteis ou até mesmo infantilizá-los, sempre que possível tentar manter a autonomia, levá-los para tomar banho de sol proporcionar jogos, brincadeiras, deixe claro que essa fase é passageira e que o isolamento é necessário.

\section{CONCLUSÃO}

Logo o estado de saúde mental dos idosos durante o período de pandemia, acabou gerando impactos de forma explícita, ponderando fatores importantes e todas as suas implicações que estavam relacionadas em relação as emergências no contexto em saúde mental, de formas favoráveis e desfavoráveis.

As recomendações das autoridades sa- nitárias é manter o distanciamento social, porém fica evidente a necessidade de promoção para intervenções e práticas psicológicas afim de minimizar os impactos negativos para a saúde mental dos idosos durante a pandemia. No entanto, o isolamento pode causar algumas consequências negativas e para quem já sofre de um transtorno mental, como depressão, o confinamento pode facilitar o agravamento do quadro.

Pessoas idosas de forma geral acabam sofrendo por medo de si mesmo, e de seus parentes que na maioria das vezes costumam estar mais próximos, podendo assim alterar fisiologicamente o apetite, o sono, o humor, e ainda causar alterações em doenças crônicas já pré-existentes como a hipertensão e diabetes muito comum nesse público por exemplo. É de suma importância reconhecer a existência de todos esses problemas e que a falta de conhecimento gerontológico e saúde mental os agravam.

Portanto, esforços imediatos devem ser empregados, em todos os níveis e pelas mais diversas áreas de conhecimento, a fim de minimizar resultados ainda mais negativos na saúde mental da população idosa.

Cabe, enfim, investir em adequada assistência à saúde e, sobretudo, na ciência em geral, para que esse período seja abreviado e todos consigam superar os desafios do cuidado para um despertar sem sentimentos de solidão, estresse, ansiedade, tristeza e até o suicídio.

\section{Referências}

1. Wang, C.et al. Immediate psychological responses and associated factors during the initial stage of the 2019 coronavirus disease (COVID-19) epidemic among the general population in china. International Journal of Environmental Research and Public Health, 17(5), 1729. Acessado em 03 de junho de 2020. Disponível em: https://doi.org/10.3390/ijerph17051729.

2. Clarfield et al. Israel Ad Hoc COVID-19 Committee: Guidelines for Care of Older Persons During a Pandemic. The American Geriatrics Society. MONTH 2020VOL. 00. Acessado em 04 de junho de 2020. Disponivel em: https://onlinelibrary. wiley.com/doi/10.1111/jgs.16554.

3. Cordeiro, Renata Cavalcanti et al . Perfil de saúde mental de idosos comunitários: um estudo transversal. Rev. Bras. Enferm., Brasília, v. 73, n. 1, e20180191, 2020 Disponivel em <http://www.scielo.br/scielo.php?script=sci_arttext\&pi$\mathrm{d}=\$ 003471672020000100172 \&$ lng=pt\&nrm=iso $>$. acessos em 04 jun. 2020. 4. Fernandes, M. G. M; GARCIA, L. G. 0 sentido da velhice para homens e muIheres idosos. Saúde Soc. São Paulo, v. 19, n. 4, p. 771-783, 2010. Disponível em: http://www.scielo.br/pdf/sausoc/v19n4/05.pdf. Acesso em: 04 de junho de 2020. 5. Brooks, S. K. et al. The psychological impact of quarantine and how to reduce it: Rapid review of the evidence. The Lancet, 395(10227), 912-920. Acessado em 04 de junho de 2020. Disponível em: https://www.thelancet.com/journals/
lancet/article/PIIS0140-6736(20)30460-8/fulltext.

6. Hao, Gang et al. Social participation and perceived depression among elderly population in South Africa. Clinical Interventions In Aging, [s.l.], v. 12, p.971-976, jun. 2017. Acessado em 04 de junho de 2020. Disponivel em: https://www.ncbi. nlm.nih.gov/pmc/articles/PMC5491569/.

7. Mendes, K.S; SILVEIRA, R. C.; GALVAO, C. M. Revisão integrativa: método de pesquisa para a incorporação de evidências na saúde e na enfermagem. Texto contexto - enferm., Florianópolis, v. 17, n. 4, p. 758-764, Dec. 2008. Acesso em 01 de junho de 2020. Disponível em: http://www.scielo.br/pdf/ tce/v17n4/18.pdf.

8. Meneguci Joilson, Meneguci Cíntia Aparecida Garcia, Moreira Marlon Martins, Pereira Kariny Rodrigues, Tribess Sheilla, Sasaki Jeffer Eidi et al . Prevalência de sintomatologia depressiva em idosos brasileiros: uma revisão sistemática com metanálise. J. bras. psiquiatr. [Internet]. 2019 Dec [cited 2020 July 26]; 68(4): 221-230. Available from: http://www.scielo.br/scielo.php?script=sci_arttext\&pid=S004720852019000400221\&lng=en. Epub Feb 14, 2020.

9. KEMENESI G. et al. Nursing homes and the elderly regarding the COVID-19 pandemic: situation report from Hungary. GeroScience 18 de May 2020. Acessado em: 02 de junho de 2020. 